\title{
HIGH ORDER ACCURATE SOLUTION OF THE INCOMPRESSIBLE NAVIER-STOKES EQUATIONS
}

\author{
ARNIM BRÜGER ${ }^{1}$ BERTIL GUSTAFSSON ${ }^{2}$ \\ PER LÖTSTEDT ${ }^{2}$ JONAS NILSSON ${ }^{2}$ \\ ${ }^{1}$ Dept of Mechanics, Royal Institute of Technology, SE-10044 Stockholm, Sweden. \\ ${ }^{2}$ Dept of Information Technology, Scientific Computing, Uppsala University, P. O. Box 337, \\ SE-75105 Uppsala, Sweden.
}

\begin{abstract}
High order methods are of great interest in the study of turbulent flows in complex geometries by means of direct simulation. With this goal in mind, the incompressible Navier-Stokes equations are discretized in space by a compact fourth order finite difference method on a staggered grid. The equations are integrated in time by a second order semi-implicit method. Stable boundary conditions are implemented and the grid is allowed to be curvilinear in two space dimensions. In every time step, a system of linear equations is solved for the velocity and the pressure by an outer and an inner iteration with preconditioning. The convergence properties of the iterative method are analyzed. The order of accuracy of the method is demonstrated in numerical experiments. The method is used to compute the flow in a channel, the driven cavity and a constricted channel.
\end{abstract}

Keywords: finite difference method, high order, incompressible flow, iterative solution

AMS subject classification 2000: 65N50, 76M12

\section{Introduction}

Spectral and pseudospectral methods are accurate methods for direct numerical simulation of turbulent flow governed by the incompressible Navier-Stokes equations. The disadvantage with these methods is that they are restricted to simple geometries such as channels. Finite difference methods of high order do not have this restriction and are almost as accurate as a spectral method. In this paper we develop such a method of fourth order accuracy in space and second order in time for two-dimensional problems. The level of the discretization error is the important issue, not so much the order of accuracy, but a certain error level is more 
easily obtained with a high order method. The same accuracy is achieved with fewer grid points or the solution has better accuracy on the same grid compared to a second order method. The solution in curvilinear body-fitted coordinates is obtained by a mapping of the equations to a rectangular grid where the derivatives are approximated. The solution is computed in the primitive variables defined on a staggered grid with local velocity components to avoid spurious oscillations. The method will be extended to the third dimension by a spectral representation aiming at direct numerical simulation of turbulent flow. High order accuracy in the spatial approximations is also necessary for large eddy simulation in order not to interfere with the subgrid model with terms proportional to the square of the grid size [16].

Let $u$ and $v$ be the velocity components in the $x$ - and $y$-direction, respectively, $p$ the pressure, and $\nu$ the kinematic viscosity. The Reynolds number is defined by $R e=u_{b} \ell / \nu$ for some characteristic velocity $u_{b}$ and length scale $\ell$. By defining $\mathbf{w}=(u v)^{T}$, differentiation with respect to an independent variable, such as time $t$, by a subscript, and the nonlinear and linear terms

$$
\mathcal{N}(\mathbf{w})=(\mathbf{w} \cdot \nabla) \mathbf{w}, \quad \mathcal{L}(\mathbf{w}, p)=\nabla p-R e^{-1} \Delta \mathbf{w},
$$

the Navier-Stokes equations in two dimensions are

$$
\begin{aligned}
\mathbf{w}_{t}+\mathcal{N}(\mathbf{w})+\mathcal{L}(\mathbf{w}, p) & =0, \\
\nabla \cdot \mathbf{w} & =0 .
\end{aligned}
$$

The space discretization of $\mathcal{N}$ and $\mathcal{L}$ in (1) is in compact form [26] and the equations are integrated in time by a semi-implicit method. The approximations of the first and second derivatives are defined implicitly and they satisfy systems of linear equations with a tridiagonal system matrix. The number of terms in the computational domain is reduced by orthogonal grids in the physical domain. The nonlinear convection term $\mathcal{N}$ is extrapolated from the previous time steps. The linear part of the discretized momentum equations $\mathcal{L}$ and the discretized divergence equation (2) are solved simultaneously at the new time level. A system of linear equations has to be solved for $\mathbf{w}$ and $p$ in every time step. The solution is computed by an iterative method until convergence is reached. The method is shown to be of fourth order in space and of second order in time in $\mathbf{w}$ and $p$ in numerical experiments. The method is analyzed with respect to boundary conditions and stability in [19], [20], [25], and [34]. Other examples of the accuracy and performance of the method are found in [4], [5]. There are stability constraints on the the timestep $\Delta t$ due to the semi-implicit time integration. Other constraints on $\Delta t$ are introduced by accuracy requirements and the convergence of the iterative solvers. It is shown in [8] that an implicit method is faster than a semi-implicit method for fully developed turbulent flow in a boundary layer but the CFL-number for good accuracy is rather low, $0.5-1$. In other flow regimes, 
such as transitional flow, a much smaller timestep is necessary for accuracy [14], thus reducing the time savings with an implicit method.

Direct numerical simulation of turbulent flow is reviewed in [14]. Other finite difference methods of high order for the Navier-Stokes equations are found in [2], [22], [27], [29], [32], [33], [40]. The velocity-vorticity equations are solved in [2] with a fourth order compact method and advanced in time by a semi-implicit scheme. The stencil is a wide fourth order centered difference in [22] applied to the equations (1) and in [29] the fourth order differential equation for the streamfunction is discretized by fourth order centered differences. The coupled streamfunction-vorticity equation is solved with a compact scheme in [27]. A fourth order method is developed and tested for incompressible flow in [32] and a sixth order method for compressible flow is presented in [33] on a staggered grid in a straight channel.

In the next section, the space and time discretizations are described and compared to other high order schemes. The method for computation of $\mathbf{w}$ and $p$ in every timestep is discussed in Sect. 3. The convergence of the iterative method is studied and it is compared to other similar methods. Finally, the flows in a straight channel, in a lid-driven cavity and a constricting channel with a curvilinear grid are computed. The accuracy and efficiency of the method is demonstrated in the numerical experiments.

\section{The discretization}

In this section the discrete equations in space and time are derived for a curvilinear grid. The approximations in space are compact and of fourth order accuracy. The solution is advanced in time by a method where the space operator is split so that the linear term in (1) and the divergence relation (2) are treated implicitly and the nonlinear term is extrapolated. Such methods are reviewed in [23] and [42].

\subsection{Time discretization}

The equations (1) and (2) are discretized in time by extrapolating $\mathcal{N}(\mathbf{w})$ from time $t^{n-r+1}$ up to $t^{n}$ with an $r$ :th order formula to $t^{n+1}$ and applying the $r$ :th order backward differentiation formula (BDF-r) [21] to $\mathbf{w}_{t}$ and $\mathcal{L}(\mathbf{w}, p)$ at $t^{n+1}$ to obtain

$$
\sum_{j=0}^{r} \alpha_{j} \mathbf{w}^{n+1-j}+\Delta t \mathcal{L}\left(\mathbf{w}^{n+1}, p^{n+1}\right)=-\Delta t \sum_{j=1}^{r} \beta_{j} \mathcal{N}\left(\mathbf{w}^{n+1-j}\right) .
$$


The coefficients $\alpha_{j}$ are such that the sum approximates $\mathbf{w}_{t}$ at $t^{n+1}$ and the $\beta$ coefficients are defined by

$$
\mathbf{w}^{n+1}=\sum_{j=1}^{r} \beta_{j} \mathbf{w}^{n+1-j}+\mathcal{O}\left(\Delta t^{r}\right),
$$

cf. [23]. The time step $\Delta t=t^{n+1}-t^{n}$ is constant in the interval of interest. In addition to (3), $\mathbf{w}^{n+1}$ satisfies the discretization of the divergence equation in (2). The result is a linear system of equations to solve for $\mathbf{w}^{n+1}$ and $p^{n+1}$ in every time step. We have implemented the second order method with $r=2$ implying $\alpha_{0}=3 / 2, \alpha_{1}=-2, \alpha_{2}=1 / 2, \beta_{1}=2, \beta_{2}=-1$. The integration can be started at $t^{0}$ with $r=1$.

The explicit part of the integration introduces restrictions on the the timestep for stability. This is analyzed in [18] assuming Oseen flow with periodic boundary conditions.

A different class of time discretizations is derived from integrating (1) in time

$$
\mathbf{w}^{n+1}-\mathbf{w}^{n}=-\int_{t^{n}}^{t^{n+1}} \mathcal{N}(\mathbf{w}) d t-\int_{t^{n}}^{t^{n+1}} \mathcal{L}(\mathbf{w}, p) d t .
$$

The first integral is then approximated by an explicit Adams-Bashforth method and the second integral by an implicit Adams-Moulton method [21]. The continuity equation is simultaneously satisfied by $\mathbf{w}^{n+1}$. A common combination is the Adams-Bashforth method of second order and the implicit trapezoidal (or Crank-Nicolson) method [24], [28], [37]. The Crank-Nicolson scheme is combined with an explicit Runge-Kutta method in [2].

\subsection{Space discretization}

To be able to solve the Navier-Stokes equations in more complicated geometries than a straight channel with a Cartesian grid, the equations are mapped from computational space $(\xi, \eta)$ into physical space $(x, y)$ by a univalent right hand sided orthogonal transformation

$$
x=x(\xi, \eta), y=y(\xi, \eta) .
$$

The topology of the physical domain $\Omega$ is assumed to be like a channel: an upper and a lower wall with no-slip boundary conditions, an inflow boundary to the left and an outflow boundary to the right or with no-slip conditions on all walls. The computational domain is a rectangle. 


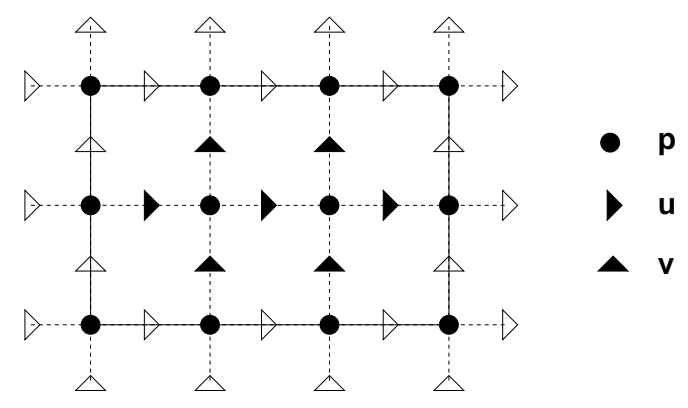

Figure 1: The staggered grid. The boundary is marked by a solid line, the inner points in the grid have filled symbols, and variables at the empty symbols are determined by the boundary conditions.

Define $\tilde{u}$ and $\tilde{v}$ to be the local velocity components in the coordinate directions $\xi$ and $\eta$, respectively, and for consistency $\tilde{p}=\tilde{p}(\xi, \eta)=p(x(\xi, \eta), y(\xi, \eta))$. The transformed system of partial differential equations is discretized on a staggered grid with grid size $(\Delta \xi, \Delta \eta)$. The velocity components $\tilde{u}$ and $\tilde{v}$ are assigned at the locations $\left(\xi_{i-\frac{1}{2} \Delta \xi}, \eta_{j}\right)$ and $\left(\xi_{i}, \eta_{j-\frac{1}{2} \Delta \eta}\right)$, respectively, and the pressure values are assigned at a grid point $\left(\xi_{i}, \eta_{j}\right)$ in the usual manner, see Fig. 1.

The reason for using locally defined velocity components on staggered orthogonal grids is that no parasitic odd-even oscillatory solutions appear, no artificial viscosity is needed, and ghost values outside $\Omega$ are required for only one velocity component at each boundary, see Fig. 1. Furthermore, with this formulation the structure of the equations is similar to the Cartesian case, especially for the gradient term $\nabla p$ and the divergence $\nabla \cdot \mathbf{w}$. They are

$$
\nabla p=\left[\begin{array}{c}
n_{1}^{-1} \tilde{p}_{\xi}, \\
n_{2}^{-1} \tilde{p}_{\eta}
\end{array}\right], \quad \nabla \cdot \mathbf{w}=\frac{1}{n_{1} n_{2}}\left(\frac{\partial}{\partial \xi}\left(n_{2} \tilde{u}\right)+\frac{\partial}{\partial \eta}\left(n_{1} \tilde{v}\right)\right),
$$

where $n_{1}=\sqrt{x_{\xi}^{2}+y_{\xi}^{2}}$ and $n_{2}=\sqrt{x_{\eta}^{2}+y_{\eta}^{2}}$ are the scale factors of the transformation (5). Unfortunately, the viscous part becomes more complicated including also first derivatives.

The derivatives for the gradient and the divergence operators (6) are centered in the middle between two variable values, while all other derivatives are centered at the grid points. Therefore, we define two different fourth order compact operators, see [13], [26], one for regular grids (7) and one for staggered grids (8). Compact operators have the advantage of smaller error constant in the truncation error and fewer numerical boundary conditions are required. For a function $f$ and grid spacing $\Delta \xi$, we have the formulas

$$
\begin{aligned}
\frac{1}{6} f_{i-1}^{\prime}+\frac{2}{3} f_{i}^{\prime}+\frac{1}{6} f_{i+1}^{\prime} & =\frac{1}{2 \Delta \xi}\left(f_{i+1}-f_{i-1}\right), \\
\frac{1}{24} f_{i-1}^{\prime}+\frac{11}{12} f_{i}^{\prime}+\frac{1}{24} f_{i+1}^{\prime} & =\frac{1}{\Delta \xi}\left(f_{i+1 / 2}-f_{i-1 / 2}\right) .
\end{aligned}
$$


In order to solve for the unknowns $f^{\prime}$, we need closed systems of the form $P f^{\prime}=Q f$ for the regular grids and $R f^{\prime}=S f$ for the staggered grids. No boundary conditions are available for the derivatives, and we use one-sided formulas for them. For the function itself on the right hand side of (7) and (8), we could use the physical boundary conditions. However, it is convenient to define $P, Q, R, S$ independently of the particular problem, and therefore we use one-sided formulas also for $f$, see [4], [5], [20], [34].

Extra indices $\xi$ and $\eta$ on the operators are introduced to indicate the coordinate direction. These formulas are one-dimensional, and are applied for all grid lines in the $\xi$ - or the $\eta$-direction. Since $\tilde{u}$ and $\tilde{v}$ are not stored at the same points, fourth order averaging formulas $E \tilde{u}$ and $E \tilde{v}$ are required for the advection terms. Let $\tilde{w}=(\tilde{u}, \tilde{v})$. Then the complete discrete equations are

$$
\begin{gathered}
\alpha_{0} \tilde{u}^{n+1}+\Delta t\left(\frac{1}{n_{1}} R_{\xi}^{-1} S_{\xi} \tilde{p}^{n+1}-R e^{-1} L_{\xi}\left(\tilde{w}^{n+1}\right)\right)= \\
-\sum_{j=1}^{q} \alpha_{j} \tilde{u}^{n+1-j}-\Delta t \sum_{j=1}^{q} \beta_{j} N_{\xi}\left(\tilde{w}^{n+1-j}\right), \\
\alpha_{0} \tilde{v}^{n+1}+\Delta t\left(\frac{1}{n_{2}} R_{\eta}^{-1} S_{\eta} \tilde{p}^{n+1}-R e^{-1} L_{\eta}\left(\tilde{w}^{n+1}\right)\right)= \\
-\sum_{j=1}^{q} \alpha_{j} \tilde{v}^{n+1-j}-\Delta t \sum_{j=1}^{q} \beta_{j} N_{\eta}\left(\tilde{w}^{n+1-j}\right), \\
\frac{1}{n_{1} n_{2}}\left(R_{\xi}^{-1} S_{\xi}\left(n_{2} \tilde{u}^{n+1}\right)+R_{\eta}^{-1} S_{\eta}\left(n_{1} \tilde{v}^{n+1}\right)\right)=0 .
\end{gathered}
$$

The Laplace operators $L_{\xi}$ and $L_{\eta}$ are

$$
\begin{aligned}
L_{\xi}(\tilde{w}) & =\frac{1}{n_{1} n_{2}}\left(P_{\xi}^{-1} Q_{\xi}\left(\frac{n_{2}}{n_{1}} P_{\xi}^{-1} Q_{\xi} \tilde{u}\right)+P_{\eta}^{-1} Q_{\eta}\left(\frac{n_{1}}{n_{2}} P_{\eta}^{-1} Q_{\eta} \tilde{u}\right)-\frac{n_{1, \eta}^{2}+n_{2, \xi}^{2}}{n_{1} n_{2}} \tilde{u}\right. \\
& \left.+\frac{2 n n_{1, \eta}}{n_{1}} P_{\xi}^{-1} Q_{\xi} E \tilde{v}-\frac{2 n_{2, \xi}}{n_{2}} P_{\eta}^{-1} Q_{\eta} E \tilde{v}+P_{\xi}^{-1} Q_{\xi}\left(\frac{n_{1, \eta}}{n_{1}}-\frac{n_{2, \eta}}{n_{2}}\right) E \tilde{v}\right), \\
L_{\eta}(\tilde{w}) & =\frac{1}{n_{1} n_{2}}\left(P_{\xi}^{-1} Q_{\xi}\left(\frac{n_{2}}{n_{1}} P_{\xi}^{-1} Q_{\xi} \tilde{v}\right)+P_{\eta}^{-1} Q_{\eta}\left(\frac{n_{1}}{n_{2}} P_{\eta}^{-1} Q_{\eta} \tilde{v}\right)-\frac{n_{1, \eta}+n_{2, \xi}^{2}}{n_{1} n_{2}} \tilde{v}\right. \\
& \left.-\frac{2 n_{1, \eta}}{n_{1}} P_{\xi}^{-1} Q_{\xi} E \tilde{u}+\frac{2 n_{2, \xi}}{n_{2}} P_{\eta}^{-1} Q_{\eta} E \tilde{u}-P_{\xi}^{-1} Q_{\xi}\left(\frac{n_{1, \eta}}{n_{1}}-\frac{n_{2, \eta}}{n_{2}}\right) E \tilde{u}\right),
\end{aligned}
$$

and the non-linear terms are

$$
\begin{aligned}
& N_{\xi}(\tilde{w})=\tilde{u}\left(\frac{1}{n_{1}} P_{\xi}^{-1} Q_{\xi} \tilde{u}+\frac{n_{1, \eta}}{n_{1} n_{2}} E \tilde{v}\right)+E \tilde{v}\left(\frac{1}{n_{2}} P_{\eta}^{-1} Q_{\eta} \tilde{u}-\frac{n_{2, \xi}}{n_{1} n_{2}} E \tilde{v}\right), \\
& N_{\eta}(\tilde{w})=E \tilde{u}\left(\frac{1}{n_{1}} P_{\xi}^{-1} Q_{\xi} \tilde{v}-\frac{n_{1, \eta}}{n_{1} n_{2}} E \tilde{u}\right)+\tilde{v}\left(\frac{1}{n_{2}} P_{\eta}^{-1} Q_{\eta} \tilde{v}+\frac{n_{2, \xi}}{n_{1} n_{2}} E \tilde{u}\right)
\end{aligned}
$$

and $n_{i, \xi}, n_{i, \eta}, i=1,2$, are derivatives of the scale factors with respect to $\xi$ and $\eta$. They are evaluated with fourth order accurate numerical differentiation. 


\subsection{Boundary conditions}

Stable boundary conditions for the Stokes and the linearized Navier-Stokes equations on staggered grids have been developed in [20], [25], [34]. We apply these conditions to our Navier-Stokes solver in the domain $\Omega$. At solid walls the velocity satisfies the no-slip condition. No numerical boundary condition is necessary for $p$.

Suppose that $\Omega$ has an inflow boundary at $x=x_{i n}$ for $y \in\left[y_{i n 1}, y_{i n 2}\right]$ and an outflow boundary at $x=x_{\text {out }}$ for $y \in\left[y_{\text {out } 1}, y_{\text {out } 2}\right]$. With Dirichlet boundary conditions at the inflow and the outflow, the set of boundary conditions in the continuous formulation in a Cartesian coordinate system is

$$
\begin{array}{rlrl}
u\left(x_{i n}, y\right)-\frac{1}{l_{\text {in }}} \int_{y_{\text {in } 1}}^{y_{\text {in } 2}} u\left(x_{\text {in }}, y\right) d y & =w_{\text {in }}(y), & u\left(x_{\text {out }}, y\right) & =u_{\text {out }}(y), \\
v\left(x_{i n}, y\right) & =v_{\text {in }}(y), & v\left(x_{\text {out }}, y\right) & =v_{\text {out }}(y), \\
\int_{y_{\text {in } 1}}^{y_{\text {in } 2}} p\left(x_{i n}, y\right) d y+\int_{y_{\text {in } 1}}^{y_{i n 2}} u\left(x_{\text {in }}, y\right) d y & =p_{\text {in }}, &
\end{array}
$$

where $\int_{y_{i n 1}}^{y_{i n 2}} w_{i n}(y) d y=0$ and $l_{i n}=y_{i n 2}-y_{i n 1}$. The velocity profiles at the inlet and the outlet are given by $\left(u_{\text {in }}, v_{\text {in }}\right)$ and $\left(u_{\text {out }}, v_{\text {out }}\right)$ and $p_{\text {in }}$ is a constant. In the discretization, the last condition in (11) replaces the first condition at $x_{i n}$ at one grid point of the inflow boundary. This point is usually chosen as the midpoint but other points also work well.

An alternative at the outflow boundary is to prescribe a zero streamwise derivative of the velocity, i.e. a Neumann condition (cf. [2]). Then the continuous conditions are

$$
\begin{aligned}
& u\left(x_{\text {in }}, y\right)=u_{\text {in }}(y), \quad \frac{\partial u\left(x_{\text {out }}, y\right)}{\partial x}-\frac{1}{l_{\text {out }}} \int_{y_{\text {out } 1}}^{y_{\text {out } 2}} \frac{\partial u\left(x_{\text {out }}, y\right)}{\partial x} d y=0, \\
& v\left(x_{i n}, y\right)=v_{\text {in }}(y), \quad \frac{\partial v\left(x_{\text {out }}, y\right)}{\partial x}=0, \\
& \int_{y_{\text {out } 1}}^{y_{\text {out } 2}} p\left(x_{\text {out }}, y\right) d y+\int_{y_{\text {out } 1}}^{y_{\text {out } 2}} \frac{\partial u\left(x_{\text {out }}, y\right)}{\partial x} d y=p_{\text {out }},
\end{aligned}
$$

where $p_{\text {out }}$ is an arbitrary constant and $l_{\text {out }}=y_{\text {out } 2}-y_{\text {out } 1}$. Also here the last condition replaces the first condition at $x_{\text {out }}$ in the midpoint of the discrete implementation. The well-posedness of the boundary conditions (11) and (12) is analyzed in [19], [25], and [34]. The pressure is well defined by the last conditions in (11) and (12) and their discretization is easily incorporated in our iterative method in the next section. Furthermore, the system matrix of the discretized equations is non-singular and the equations can be solved to machine precision. 
The boundary of the domain $\Omega$ is located at the grid points where $p$ is defined, see Fig. 1. One row of extra ghost variables for one of the velocity components are defined outside $\Omega$ to simplify the application of the boundary conditions.

\section{Iterative method}

A system of linear equations has to be solved for the velocity and the pressure at the inner points of the grid and $t=t^{n+1}$ according to (9). The iterative method for solution of the system is described here and its convergence properties are analyzed. A comparison with other iteration algorithms is made. The norm in this section is the Euclidean vector norm and its subordinate spectral matrix norm.

\subsection{Algorithm}

Let $\mathbf{w}$ denote the vector of local velocity variables in $\Omega, \overline{\mathbf{w}}$ the ghost values outside $\Omega$ and on the boundary (see Fig. 1), and let $p$ be the pressure vector. Then $\mathbf{w}, \overline{\mathbf{w}}$, and $p$ satisfy a system of linear equations

$$
\left(\begin{array}{ccc}
A_{0} & A_{1} & G \\
A_{2} & C & B \\
D_{0} & D_{1} & 0
\end{array}\right)\left(\begin{array}{c}
\mathbf{w} \\
\overline{\mathbf{w}} \\
p
\end{array}\right)=\left(\begin{array}{c}
b \\
\bar{b} \\
0
\end{array}\right) .
$$

Let $L$ denote the discretization of the Laplace operator in the inner points, see (10). Then $A_{0}$ is defined by

$$
A_{0}=\alpha_{0} I-R e^{-1} \Delta t L .
$$

The part of the Laplace operator operating on the velocity components on and outside the boundary is represented by $A_{1}$. The gradient approximation $G$ has linearly dependent columns and $p$ is not uniquely defined unless $B \neq 0$. With our choice of boundary conditions in (11) and (12), $C$ is nonsingular and diagonal except for a diagonal block with non-zero elements and $B$ has rank one and one row different from zero. An LU-factorization of a small part of $C$ due to the boundary integrals is stored for use in the iterative solver. The values in the interior are coupled to the boundary values and the outer ghost values via the sparse matrix $A_{2}$. The boundary conditions supported by a stability analysis define $A_{2}, B$, and $C$. The approximation of the divergence is $\left(D_{0}, D_{1}\right)$. The matrices $A_{0}, D_{0}$, and $G$ are dense due to the implicit approximation of the derivatives and are not known explicitly. The right hand side, $b$ and $\bar{b}$, depends on old values of $\mathbf{w}$ and the boundary conditions.

After expressing $\overline{\mathbf{w}}$ in $\mathbf{w}, p$, and $\bar{b}$ and defining

$$
\begin{array}{lll}
\hat{A}=A_{0}-A_{1} C^{-1} A_{2}, & \hat{G}=G-A_{1} C^{-1} B, & c=b-A_{1} C^{-1} \bar{b}, \\
\hat{D}=D_{0}-D_{1} C^{-1} A_{2}, & \hat{B}=-D_{1} C^{-1} B, & d=-D_{1} C^{-1} \bar{b},
\end{array}
$$


the system to be solved is

$$
\left(\begin{array}{cc}
\hat{A} & \hat{G} \\
\hat{D} & \hat{B}
\end{array}\right)\left(\begin{array}{c}
\mathbf{w} \\
p
\end{array}\right)=\left(\begin{array}{c}
c \\
d
\end{array}\right)
$$

Since $A_{1} \sim h^{-2} \Delta t / R e$ and $C^{-1}$ and $A_{2}$ are of $\mathcal{O}(1), \hat{A}$ has the same form as $A_{0}$ in $(14)$

$$
\hat{A}=\alpha_{0} I-R e^{-1} \Delta t \hat{L},
$$

but now the boundary conditions are included in the discrete Laplace operator. For the gradient approximation we have $G \sim h^{-1} \Delta t$ and $\hat{G} \sim h^{-1} \Delta t$, and the divergence approximation satisifes $D_{0} \sim h^{-1}, D_{1} \sim h^{-1}$, and $\hat{D} \sim h^{-1}$, where $\Delta \xi, \Delta \eta \sim h$.

In the iterative method we propose for solving (15), only the result of a multiplication of an arbitrary vector by the matrix is needed. It follows from (9) that this operation is performed by operating with $Q, S$, or $E$ on w and $p$ and one or two solutions of a tridiagonal system defined by $P$ or $R$.

The system (15) is rewritten as

$$
M x=b,
$$

where $x^{T}=\left(x_{1}^{T}, x_{2}^{T}\right)=\left(\mathbf{w}^{T}, p^{T}\right), b^{T}=\left(b_{1}^{T}, b_{2}^{T}\right)=\left(c^{T}, d^{T}\right)$. An approximate factorization of $M$ in (17) is determined in a manner similar to [37]

$$
\tilde{M}=\left(\begin{array}{cc}
\hat{A} & 0 \\
\hat{D} & I
\end{array}\right)\left(\begin{array}{cc}
I & \alpha_{0}^{-1} \hat{G} \\
0 & -\left(\alpha_{0}^{-1} \hat{D} \hat{G}-\hat{B}\right)
\end{array}\right)=\left(\begin{array}{cc}
\hat{A} & \alpha_{0}^{-1} \hat{A} \hat{G} \\
\hat{D} & \hat{B}
\end{array}\right) .
$$

The difference between $M$ and its approximation $\tilde{M}$ is

$$
M-\tilde{M}=\left(\begin{array}{cc}
0 & \alpha_{0}^{-1} R e^{-1} \Delta t \hat{L} \hat{G} \\
0 & 0
\end{array}\right) .
$$

Suppose that $\tilde{M}$ is invertible. In an outer fixed point iteration the correction $\delta x$ is computed as

$$
x^{(k+1)}=x^{(k)}+\delta x^{(k)}=x^{(k)}+\tilde{M}^{-1}\left(b-M x^{(k)}\right)=x^{(k)}+\tilde{M}^{-1} r^{(k)} .
$$

One or two outer iterations usually suffice depending on how well $\tilde{M}$ approximates $M$.

The solution of

$$
\tilde{M} \delta x^{(k)}=r^{(k)}
$$


is computed using the factorization (18). Then with forward and back substitution we have

$$
\begin{array}{ll}
\text { 1. } & \hat{A} y_{1}=r_{1} \\
\text { 2. } & y_{2}=r_{2}-\hat{D} y_{1}, \\
\text { 3. } & \left(\alpha_{0}^{-1} \hat{D} \hat{G}-\hat{B}\right) \delta x_{2}=-y_{2}, \\
\text { 4. } & \delta x_{1}=y_{1}-\alpha_{0}^{-1} \hat{G} \delta x_{2}
\end{array}
$$

where $\delta x^{(k)}=\left(\delta x_{1}^{T}, \delta x_{2}^{T}\right)^{T}$ and $r^{(k)}=\left(r_{1}^{T}, r_{2}^{T}\right)^{T}$. If $B=0$ then $\hat{B}=0$ and $\hat{G}=G$. Since $G$ does not have full rank, $\hat{D} \hat{G}$ in (22c) is singular. This is not a problem for straight channels but for curvilinear geometries the equation has no solution. From the definition of the residual in (20) it follows that the right hand side in $(22 \mathrm{~b})$ can be written as $\hat{D} z$ for some $z$. The reason why $\hat{D} z$ is not necessarily in the range of $\hat{D} \hat{G}$ for a curvilinear channel is the difference there between $D$ and $G^{T}$ in (9) when $n_{1}, n_{2} \neq 1$. By choosing $B$ of low rank but such that $\alpha_{0}^{-1} \hat{D} \hat{G}-\hat{B}$ is nonsingular, a solution $\delta x_{2}$ is guaranteed. The singularity is removed by letting a stable choice of boundary conditions define $B$.

The first system of equations

$$
\hat{A} y_{1}=r_{1}
$$

is solved by fixed point iteration and a preconditioning matrix $\tilde{A}$ as in (20)

$$
y_{1}^{(k+1)}=y_{1}^{(k)}+\delta y_{1}^{(k)}=y_{1}^{(k)}+\tilde{A}^{-1}\left(r_{1}-\hat{A} y_{1}^{(k)}\right) .
$$

The simplest choice of $\tilde{A}$ is $\alpha_{0}^{-1} I$.

The third equation in $(22)$

$$
\left(\alpha_{0}^{-1} \hat{D} \hat{G}-\hat{B}\right) \delta x_{2}=-y_{2}
$$

is similar to the Poisson equation but the system matrix is not necessarily symmetric due to $\hat{B}$, the coordinate transformation, and boundary conditions. The matrix is not available explicitly and an iterative Krylov method [17], only requiring the computation of the matrix-vector product $\left(\alpha_{0}^{-1} \hat{D} \hat{G}-\hat{B}\right) y$ for an arbitrary $y$, is chosen for solution of (25). Bi-CGSTAB [44] is such a method suitable for nonsymmetric matrices with eigenvalues away from the imaginary axis and the equivalent of only about five solution vectors is used as workspace.

The convergence of the iterations is slow without a preconditioner of the matrix. Incomplete LU factorization (ILU) [30] is often efficient in improving the convergence rate for discretizations of elliptic equations. A disadvantage is that an ILU preconditioner may have high storage requirements depending on the chosen amount of fill-in in the algorithm. The same ILU factorization is used repeatedly in every time step since $\alpha_{0}^{-1} \hat{D} \hat{G}-\hat{B}$ is constant. It requires access to 
the matrix elements of $\alpha_{0}^{-1} \hat{D} \hat{G}-\hat{B}$ but their values are not known. Instead, the second order accurate approximations of the divergence and the gradient, $D_{2}$ and $G_{2}$, are generated and their composition $D_{2} G_{2}$. It is sparse and the ILU factors $L$ and $U$ for $\alpha_{0}^{-1} \hat{D} \hat{G}-\hat{B}$ are based on $D_{2} G_{2}$.

\subsection{Convergence analysis and termination criteria}

Assume that the equations in steps 1 and 2 in the inner iterations in (22) are solved so that the residuals are $\rho_{1}$ and $\rho_{2}$ in the $k$ :th outer iteration

$$
\left(\begin{array}{cc}
\hat{A} & 0 \\
\hat{D} & I
\end{array}\right)\left(\begin{array}{l}
y_{1} \\
y_{2}
\end{array}\right)=\left(\begin{array}{l}
r_{1}+\rho_{1} \\
r_{2}+\rho_{2}
\end{array}\right)=r^{(k)}+\rho^{(k)} .
$$

Then the recursion for the residual in (20) is

$$
\begin{aligned}
r^{(k+1)} & =b-M x^{(k+1)}=b-M x^{(k)}-M \tilde{M}^{-1}\left(r^{(k)}+\rho^{(k)}\right) \\
& =\left(I-M \tilde{M}^{-1}\right) r^{(k)}-M \tilde{M}^{-1} \rho^{(k)} .
\end{aligned}
$$

After some algebraic manipulations we find from (18) and (16) that

$$
M \tilde{M}^{-1}=\left(\begin{array}{cc}
I-F \hat{D} \hat{A}^{-1} & F \\
0 & I
\end{array}\right), F=-\alpha_{0}^{-1} R e^{-1} \Delta t \hat{L} \hat{G}\left(\alpha_{0}^{-1} \hat{D} \hat{G}-\hat{B}\right)^{-1} .
$$

A necessary condition for the iterations (27) to converge is that the spectral radius of $I-M \tilde{M}^{-1}$ is less than one, i.e. the eigenvalues of $F \hat{D} \hat{A}^{-1}$ have modulus less than one. The conclusion from $F$ in $(28)$ is that for a $R e$ and a given grid, $\Delta t$ has to be sufficiently small for the outer iterations to converge.

The expression for the residual in (27) is

$$
r^{(k+1)}=\left(I-M \tilde{M}^{-1}\right)^{k+1} r^{(0)}-\sum_{j=0}^{k}\left(I-M \tilde{M}^{-1}\right)^{j} M \tilde{M}^{-1} \rho^{(k-j)},
$$

and if $\left\|I-M \tilde{M}^{-1}\right\| \leq \mu$ the termination criterion for the inner iterations (26) is $\left\|\rho^{(j)}\right\| \leq \varepsilon_{i}$ then

$$
\left\|r^{(k+1)}\right\| \leq \mu^{k+1}\left\|r^{(0)}\right\|+\frac{(1+\mu)\left(1-\mu^{k+1}\right)}{1-\mu} \varepsilon_{i} .
$$

A sufficient condition for convergence is $\mu<1$. It follows from (28) that this is possible if $F$ is sufficiently small, e.g. by taking $\Delta t$ sufficiently small. Then

$$
\lim _{k \rightarrow \infty}\left\|r^{(k)}\right\|=\frac{1+\mu}{1-\mu} \varepsilon_{i}
$$


and for $r^{(k)}$ to satisfy the termination criterion for the outer iterations $\left\|r^{(k)}\right\| \leq \varepsilon_{o}$, $\varepsilon_{i}$ should be chosen

$$
\varepsilon_{i} \leq(1-\mu) \varepsilon_{o} /(1+\mu) .
$$

Since $\mu<1$ the requirements on the inner iterations are slightly more strict compared to the outer termination condition.

It follows from (22) and (26) that

$$
\rho_{1}=\hat{A} y_{1}-r_{1}, \rho_{2}=-\left(\alpha_{0}^{-1} \hat{D} \hat{G}-\hat{B}\right) \delta x_{2}-r_{2}+D y_{1} \text {. }
$$

Hence, $\rho_{1}$ and $\rho_{2}$ are the remaining residuals when the linear systems in steps 1 and 3 in (22) are solved iteratively. In order to satisfy (29) the stopping criteria in $(22)$ are $\varepsilon_{1}=\varepsilon_{3}=\varepsilon_{i} / \sqrt{2}$ so that

$$
\left\|\rho^{(k)}\right\|^{2}=\left\|\rho_{1}\right\|^{2}+\left\|\rho_{2}\right\|^{2} \leq \varepsilon_{i}^{2} \leq \varepsilon_{o}^{2}(1-\mu)^{2} /(1+\mu)^{2} .
$$

The same analysis applied to (24) shows that the iterations converge if

$$
I-\tilde{A}^{-1} \hat{A}=\alpha_{0}^{-1} R e^{-1} \Delta t \hat{L}
$$

satisfies $R e^{-1} \Delta t\|\hat{L}\|<\alpha_{0}$. The right hand side in (30) is also a multiplying factor in $F$ defined in (28). Since $\hat{L} \sim h^{-2}$, the fixed point iteration (24) converges if $h^{-2} \Delta t / R e$ is sufficiently small.

The discrete Laplacian of fourth order $\hat{D} \hat{G}$ is approximated by the corresponding $D_{2} G_{2}$ of second order when the preconditioning (here the ILU factorization) is computed. The assumption that $-\hat{D} \hat{G},-D_{2} G_{2}$, and the preconditioner are symmetric and positive definite and Fourier analysis give a clue why this preconditioner has an effect on the convergence rate of the iterative solution in (22c).

A rectangular physical domain with periodic boundary conditions is discretized with a Cartesian grid with constant step sizes $\Delta x$ and $\Delta y$. Let $\omega_{1}$ and $\omega_{2}$ be the discrete wavenumbers in the $x$ and $y$ directions and introduce the notation

$$
\begin{aligned}
& \varphi_{1}=\omega_{1} \Delta x, \varphi_{2}=\omega_{2} \Delta y, 0 \leq\left|\varphi_{1}\right|,\left|\varphi_{2}\right| \leq \pi \\
& \kappa_{1}=\Delta t / \Delta x, \quad \kappa_{2}=\Delta t / \Delta y, s_{j}=\sin \varphi_{j} / 2, c_{j}=\cos \varphi_{j} / 2, j=1,2 .
\end{aligned}
$$

For the Fourier transformation of the equations we need the following coefficients

$$
b_{1}=\frac{24 i s_{1}}{11+\cos \varphi_{1}}=\frac{12 i s_{1}}{5+c_{1}^{2}}, b_{2}=\frac{24 i s_{2}}{11+\cos \varphi_{2}}=\frac{12 i s_{2}}{5+c_{2}^{2}} .
$$

The symbol of $\hat{D} \hat{G}$ is (cf. [18])

$$
\mu_{\hat{D} \hat{G}}=\kappa_{1} b_{1}^{2}+\kappa_{2} b_{2}^{2}=-144\left(\frac{\kappa_{1} s_{1}^{2}}{\left(5+c_{1}^{2}\right)^{2}}+\frac{\kappa_{2} s_{2}^{2}}{\left(5+c_{2}^{2}\right)^{2}}\right) .
$$


The second order approximation $D_{2} G_{2}$ has the symbol

$$
\mu_{D_{2} G_{2}}=-4\left(\kappa_{1} s_{1}^{2}+\kappa_{2} s_{2}^{2}\right) .
$$

Since

$$
\frac{36}{25} \mu_{D_{2} G_{2}}=-\frac{144}{25}\left(\kappa_{1} s_{1}^{2}+\kappa_{2} s_{2}^{2}\right) \leq \mu_{D G} \leq-4\left(\kappa_{1} s_{1}^{2}+\kappa_{2} s_{2}^{2}\right)=\mu_{D_{2} G_{2}},
$$

the two operators are spectrally equivalent.

Suppose that $H, K$, and $L$ are symmetric and positive definite and that $\lambda_{j}(C)$ is the $j$ :th eigenvalue of $C$. Then one can show using Corr. 3.14 in [1] that

$$
\lambda_{j}\left(H^{-1} L\right) \lambda_{\max }\left(L^{-1} K\right) \geq \lambda_{j}\left(H^{-1} K\right) \geq \lambda_{j}\left(H^{-1} L\right) \lambda_{\min }\left(L^{-1} K\right) .
$$

For the quotient $\lambda_{\max } / \lambda_{\min }$ for $H^{-1} K$ we have

$$
\frac{\lambda_{\max }\left(H^{-1} L\right) \lambda_{\max }\left(L^{-1} K\right)}{\lambda_{\min }\left(H^{-1} L\right) \lambda_{\min }\left(L^{-1} K\right)} \geq \frac{\lambda_{\max }\left(H^{-1} K\right)}{\lambda_{\min }\left(H^{-1} K\right)} \geq \frac{\lambda_{\max }\left(H^{-1} L\right) \lambda_{\min }\left(L^{-1} K\right)}{\lambda_{\min }\left(H^{-1} L\right) \lambda_{\max }\left(L^{-1} K\right)} .
$$

Let $H$ denote the preconditioning matrix, $L=-D_{2} G_{2}$, and $K=-\hat{D} \hat{G}$ in (32). The convergence rate of an iterative method usually improves when $\lambda_{\max } / \lambda_{\min }$ is small for the system matrix. Without preconditioning $\lambda_{\max }(-\hat{D} \hat{G}) / \lambda_{\min }(-\hat{D} \hat{G})$ is of $\mathcal{O}\left(h^{-2}\right)$. With a suitable preconditioner $H$ such as a modified ILU factorization, see [1], [17], $\lambda_{\max }\left(-H^{-1} D_{2} G_{2}\right) / \lambda_{\min }\left(-H^{-1} D_{2} G_{2}\right)$ is of $\mathcal{O}\left(h^{-1}\right)$. Since $D_{2} G_{2}$ and $\hat{D} \hat{G}$ are spectrally equivalent according to (31) and

$\lambda_{\text {max }}\left(\left(D_{2} G_{2}\right)^{-1} \hat{D} \hat{G}\right) / \lambda_{\text {min }}\left(\left(D_{2} G_{2}\right)^{-1} \hat{D} \hat{G}\right)$ is of $\mathcal{O}(1)$, we have from (32) that also $\lambda_{\text {max }}\left(-H^{-1} \hat{D} \hat{G}\right) / \lambda_{\min }\left(-H^{-1} \hat{D} \hat{G}\right)$ is of $\mathcal{O}\left(h^{-1}\right)$.

\subsection{Comparison with other approaches}

In a fractional step method [3], [24], [41], the momentum equation is first advanced and then a correction to the velocity is introduced so that the divergence condition is satisfied. These are the steps also in (22). First, the implicit part of the momentum equation is solved for $y_{1}$. Then a Poisson-like equation is solved for a pressure correction so that the continuity equation is satisfied. Finally, the velocity correction is updated. The SIMPLE and SIMPLER algorithms are also of this form, see [36]. The boundary conditions of the intermediate variables cause no problems here as they do in [3] and [41], since they are defined by the matrices in the semi-implicit discretization. The fractional step method is simliar to a projection method [3] where a provisional $\mathbf{w}$ is computed and then projected into the divergence free subspace. Instead of solving the continuity equation (2), an elliptic equation for $p$ can be derived. How to construct accurate and stable boundary conditions for $p$ in a second order method is investigated in [38]. These difficulties are avoided in our approach. 
An alternative to the approximate factorization in (18) is to solve for $\mathbf{w}$ in (15) and then insert $\mathbf{w}$ into the continuity equation to arrive at

$$
\left(\hat{D} \hat{A}^{-1} \hat{G}-\hat{B}\right) p=\hat{D} \hat{A}^{-1} c-d,
$$

followed by

$$
\mathbf{w}=\hat{A}^{-1}(c-\hat{G} p) .
$$

This idea can be viewed as the result of an exact factorization of $M$ in (17), where the left hand side matrix in (33) is the Schur complement. An outer iteration is not employed here but in our iterative method (22) only one outer iteration is needed in most cases to satisfy the convergence criterion (see Fig. 9 in Sect. 4). In an iterative solution of (33), the inverse of $A$ is a dense matrix and expensive to compute. Therefore, $\hat{A}^{-1}$ in (33) and (34) is usually approximated by some $\tilde{A}^{-1}$ which is easy to compute. In (25), $\tilde{A}^{-1}=\alpha_{0}^{-1} I$ and other alternatives including different approximations of $\hat{A}^{-1}$ in (33) and (34) for second order time accuracy are found in [7], [9] and [35]. The Schur complement in (33) is approximated in [43] yielding an approximate factorization of $M$ different from (18). With approximations of $\hat{A}^{-1}$ and outer iterations in (33) and (34) the algorithm is of Uzawa type [6], [37].

For rapid solution of (17) and (25) a method accelerating the convergence is needed. An overview of iterative methods and preconditioners for (17) is found in [10] and the convergence of a particular preconditioning operator is analyzed in [12] and applied in [11]. The multigrid method is applied to the solution of the incompressible Navier-Stokes equations in [2], [15], [31], [36]. A possibility is to apply the multigrid algorithm only to (25) instead of the ILU preconditioner. Multigrid iteration is often very efficient for the Poisson equation and explicit knowledge of $\hat{D} \hat{G}$ is not necessary.

\section{Numerical results}

The spatial and temporal accuracy of the solution is verified in four different geometries with non-uniform grids. Small perturbations in the form of OrrSommerfeld eigenmodes are added to plane Poiseuille flow in a straight channel. The solution is compared to a solution obtained with a spectral method. The steady state flow in a driven cavity is calculated at high $R e$ and compared to results from [15]. Two channels with a constriction and non-Cartesian grids are the last two examples. The accuracy of the steady state solution is evaluated using a forcing function and by comparing with a fine grid solution.

\subsection{Orr-Sommerfeld modes for plane Poiseuille flow}

Eigensolutions can be derived for the linearized form of the Navier-Stokes equations in a straight 2D channel according to the Orr-Sommerfeld theory as dis- 
cussed in e.g. [39]. The analysis provides eigenmodes that are well suited for convergence tests. A time dependent reference solution of high accuracy is computed for this purpose.

For plane 2D Poiseuille flow, an eigenmode $\left(\mathbf{w}_{m}, p_{m}\right)$ is of the form

$$
\mathbf{w}_{m}(x, y, t)=\tilde{\mathbf{w}}(y) e^{i(\alpha x-\omega t)}, p_{m}(x, y, t)=\tilde{p}(y) e^{i(\alpha x-\omega t)},
$$

with the streamwise wavenumber $\alpha$ and $\omega=\alpha c$ where $c$ is the corresponding eigenvalue. The imaginary part of $c$ determines the stability of the mode. Unstable modes appear when $R e$ exceeds a critical threshold.

Since the Orr-Sommerfeld modes result from linear stability theory we introduce the mode as a perturbation to the Poiseuille base flow $U_{b}$ as

$$
U=U_{b}+\epsilon \mathbf{w}_{m}=U_{b}+\epsilon \tilde{\mathbf{w}}(y) e^{i(\alpha x-\omega t)}, U_{b}=\left(\begin{array}{c}
1-y^{2} \\
0
\end{array}\right)
$$

for a small $\epsilon$. For the pressure we have

$$
p=\epsilon p_{m}=\epsilon \tilde{p}(y) e^{i(\alpha x-\omega t)} .
$$
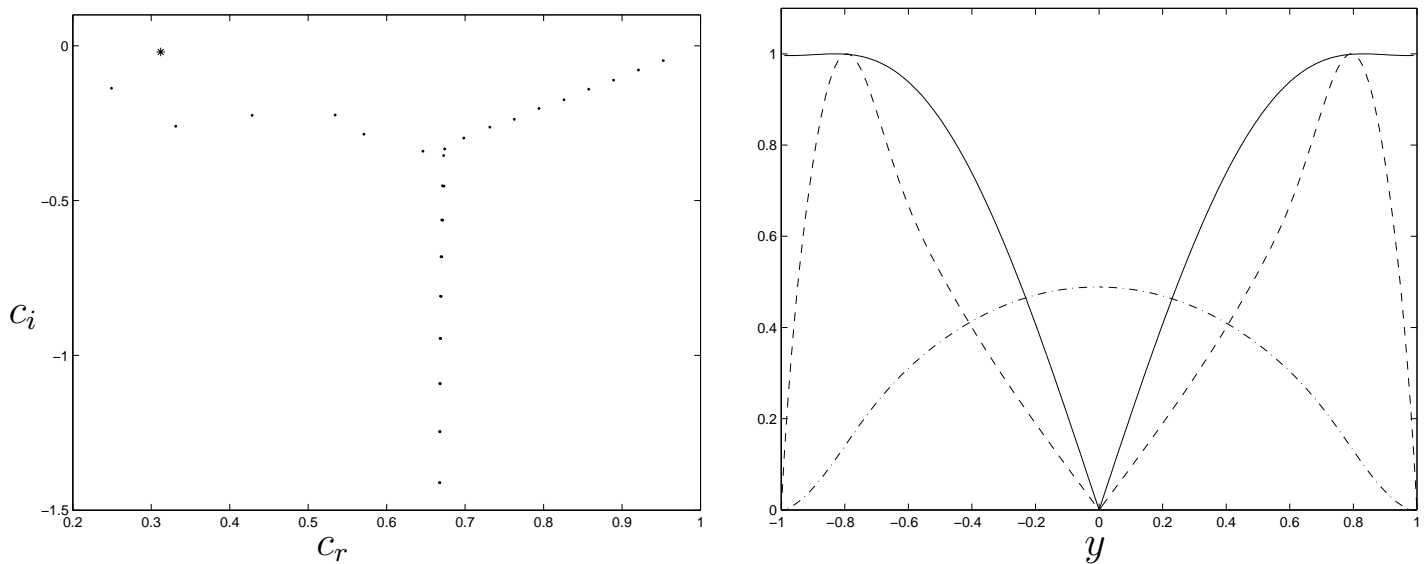

Figure 2: Eigenvalues (left) for 2D Orr-Sommerfeld modes in plane Poiseuille flow at $R e=2000, \alpha=1$. Shape (right) of the normalized, absolute eigenfunctions of velocities $u$ (dashed), $v$ (dash-dotted) and pressure (solid) of the mode corresponding to the eigenvalue marked with $*$.

The mode investigated here is the least stable one for $R e=2000$ which is below the critical Reynolds number. In the left panel of Fig. 2, the Orr-Sommerfeld modes are shown for $2 \mathrm{D}$ Poiseuille flow at $R e=2000$. In our numerical experiments the eigenmode with eigenvalue $c$ marked with $*$ is chosen. Its real and 
imaginary parts are $c_{r}=0.3121$ and $c_{i}=-0.0198$, respectively. The eigenfunctions for $u, v$ and $p$ are computed using a solver developed in [39] and can be considered to be very accurate since they are obtained in Chebyshev space expanded in a large number of modes. The shape of the specific eigenfunctions is shown to the right in Fig. 2.

The Orr-Sommerfeld solution with $\epsilon=0.1$ is compared to the solution obtained with our scheme. The parameter $\epsilon$ in (35) and (36) has to be a small in order to avoid nonlinear interactions. The computational domain is $\Omega=$ $[0,4 \pi] \times[-1,1]$ covering two streamwise wavelengths of the eigenmode. Time dependent Dirichlet boundary conditions are applied at the in- and outflow boundaries. The grid is stretched in wall normal direction by a cubic function in order to achieve proper resolution of the largest gradients in the velocities. The simulation is run with a small constant time step $\Delta t=10^{-4}$ to ensure that the numerical error from the spatial discretization dominates until time $T=10=10^{5} \Delta t$. The error is measured in the $\ell_{2}$ norm $\|\cdot\|_{2}$ and the maximum norm $\|\cdot\|_{\infty}$ defined as

$$
\|e\|_{2}^{2}=\sum_{i, j} e_{i j}^{2} / N,\|e\|_{\infty}=\max _{i, j}\left|e_{i j}\right|
$$

where $(i, j)$ is an index of an inner point in the grid and $N$ is the total number of inner grid points.

Let $f$ be a component of the solution determined by the Orr-Sommerfeld approximation. For grid $k, k \geq 0$, the solution is $f_{k}$ with grid size locally changing with $k$ as $2^{k} h$. Then the assumption is that the solution error $e_{k}$ behaves as

$$
e_{k}=f_{k}-f=c\left(2^{k} h\right)^{q}
$$

for some $c$ depending on $x, y$, and the solution. In order to determine spatial convergence $q$ the computation is repeated on three grid sizes $16 \times 21,31 \times 41,61 \times$ 81 with $k=2,1,0$.

Tables 1, 2, and 3 show the achieved error norms and convergence rates for the two finest grids when compared to the next coarser grid at $T=4,10$. The results are similar at $T=2,6,8$. The error norms are small over a large number of time steps and the order of accuracy is the expected one in all variables. 


\begin{tabular}{ccccc}
\hline \multirow{2}{*}{ time step } & & \multicolumn{3}{c}{$u$ error norms (q-order) } \\
\cline { 3 - 5 } & & $16 \times 21$ & $31 \times 41$ & $61 \times 81$ \\
\hline \multirow{2}{*}{40000} & $\ell_{2}$ & $1.00 e-06$ & $4.91 e-08(4.3)$ & $1.64 e-09(4.9)$ \\
& MAX & $3.58 e-06$ & $2.07 e-07(4.1)$ & $5.90 e-09(5.1)$ \\
\cline { 3 - 5 } 100000 & $\ell_{2}$ & $1.55 e-06$ & $5.73 e-08(4.8)$ & $2.09 e-09(4.8)$ \\
& MAX & $6.62 e-06$ & $1.98 e-07(5.1)$ & $8.03 e-09(4.6)$ \\
\hline
\end{tabular}

Table 1: Orr-Sommerfeld solutions for 2D Poiseuille flow. The spatial errors in $u$ measured in the $\ell_{2}$ and maximum norms and order of accuracy are shown.

\begin{tabular}{ccccc}
\hline \multirow{2}{*}{ time step } & & \multicolumn{3}{c}{$v$ error norms $(q$-order $)$} \\
\cline { 3 - 5 } & & $16 \times 21$ & $31 \times 41$ & $61 \times 81$ \\
\hline \multirow{2}{*}{40000} & $\ell_{2}$ & $1.54 e-07$ & $3.75 e-09(5.4)$ & $1.60 e-10(4.6)$ \\
& MAX & $4.52 e-07$ & $1.00 e-08(5.5)$ & $5.65 e-10(4.1)$ \\
\cline { 2 - 5 } 100000 & $\ell_{2}$ & $3.76 e-07$ & $8.21 e-09(5.5)$ & $2.16 e-10(5.2)$ \\
& MAX & $8.58 e-07$ & $2.12 e-08(5.3)$ & $9.76 e-10(4.4)$ \\
\hline
\end{tabular}

Table 2: Orr-Sommerfeld solutions for 2D Poiseuille flow. The spatial errors in $v$ measured in the $\ell_{2}$ and maximum norms and order of accuracy are shown.

\begin{tabular}{ccccc}
\hline \multirow{2}{*}{ time step } & & \multicolumn{3}{c}{$p$ error norms $(q$-order $)$} \\
\cline { 3 - 5 } & & $16 \times 21$ & $31 \times 41$ & $61 \times 81$ \\
\hline \multirow{2}{*}{40000} & $\ell_{2}$ & $1.96 e-07$ & $8.14 e-09(4.6)$ & $8.95 e-10(3.2)$ \\
& MAX & $4.08 e-07$ & $1.96 e-08(4.4)$ & $1.66 e-09(3.6)$ \\
\cline { 3 - 5 } 100000 & $\ell_{2}$ & $1.78 e-07$ & $1.03 e-08(4.1)$ & $8.02 e-10(3.7)$ \\
& MAX & $5.00 e-07$ & $2.35 e-08(4.4)$ & $1.75 e-09(3.8)$ \\
\hline
\end{tabular}

Table 3: Orr-Sommerfeld solutions for 2D Poiseuille flow. The spatial errors in $p$ measured in the $\ell_{2}$ and maximum norms and order of accuracy are shown. 

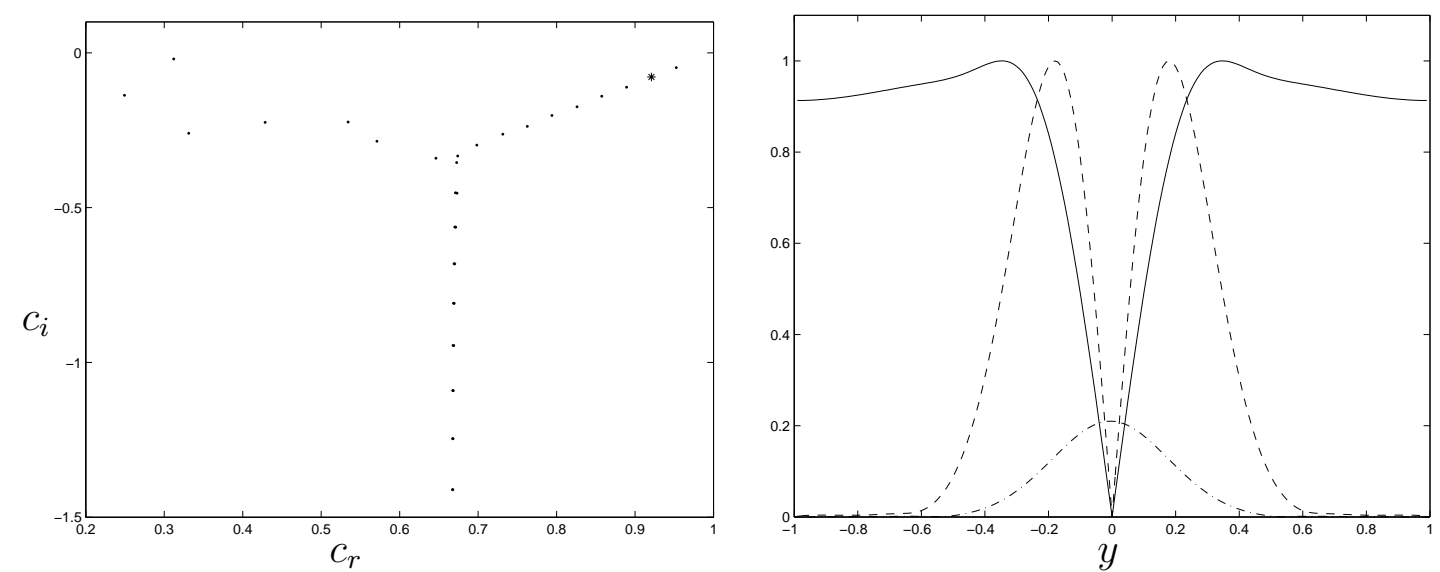

Figure 3: Eigenvalues (left) for 2D Orr-Sommerfeld modes in plane Poiseuille flow at $R e=2000, \alpha=1$. Shape (right) of normalized, absolute eigenfunctions of velocities $u$ (dashed), $v$ (dash-dotted) and pressure (solid) of the mode corresponding the eigenvalue marked with $*$.

For evaluation of the temporal error we choose an Orr-Sommerfeld mode that is propagating faster in time in order to increase the temporal error. This eigenvalue has a large real part and is marked in the eigenvalue map in the left part of Fig. 3. It is the third least stable mode for $R e=2000$ with $c_{r}=0.9209$ and $c_{i}=-0.0782$. The corresponding eigenfunctions are plotted in the right of Fig. 3.

The solution is computed with the same $\epsilon$ at $T=5$ with three different time steps on a $31 \times 41$ grid without stretching in the same channel. The time steps are large since the time error now should dominate over the error from the spatial discretization. In the solver at least four outer iterations are applied to ensure a fully converged pressure solution in each time step. Table 4 displays the errors measured as in (37) and the order of accuracy $r$ is calculated as in (38) with $h$ replaced by $\Delta t$. Second order accurate solutions are achieved in all components. Similar results are obtained at $T=2.5$. The global error grows linearly in $u$ and $v$ and is almost constant in $p$. Second order temporal accuracy in $p$ is usually not obtained with only one outer iteration or only one pressure correction per timestep.

\begin{tabular}{lccc}
\hline & $\Delta t=0.1$ & $\Delta t=0.05$ & $\Delta t=0.025$ \\
$u$ error (order) & $2.10 e-06$ & $5.46 e-07(1.9)$ & $1.46 e-07(1.9)$ \\
$v$ error (order) & $6.34 e-07$ & $1.60 e-07(2.0)$ & $3.53 e-08(2.2)$ \\
$p$ error (order) & $1.98 e-07$ & $5.28 e-08(1.9)$ & $1.18 e-08(2.2)$ \\
\hline
\end{tabular}

Table 4: Time accuracy at $T=5.0$. The temporal error is measured in the maximum norm and the order of the error is computed. 


\subsection{Driven cavity}

The Navier-Stokes equations are solved for the driven cavity problem. This is a standard problem on a two-dimensional square $[0,1] \times[0,1]$ with a Cartesian grid. The steady flow is computed in a closed cavity with a prescribed velocity at the upper wall $y=1$. The streamlines of the solution at two different $R e$ are found in Fig. 4. The steady state is reached by integrating the time-dependent equation until the time-derivatives are sufficiently small. Only one outer iteration in the iterative method is needed in the time steps.
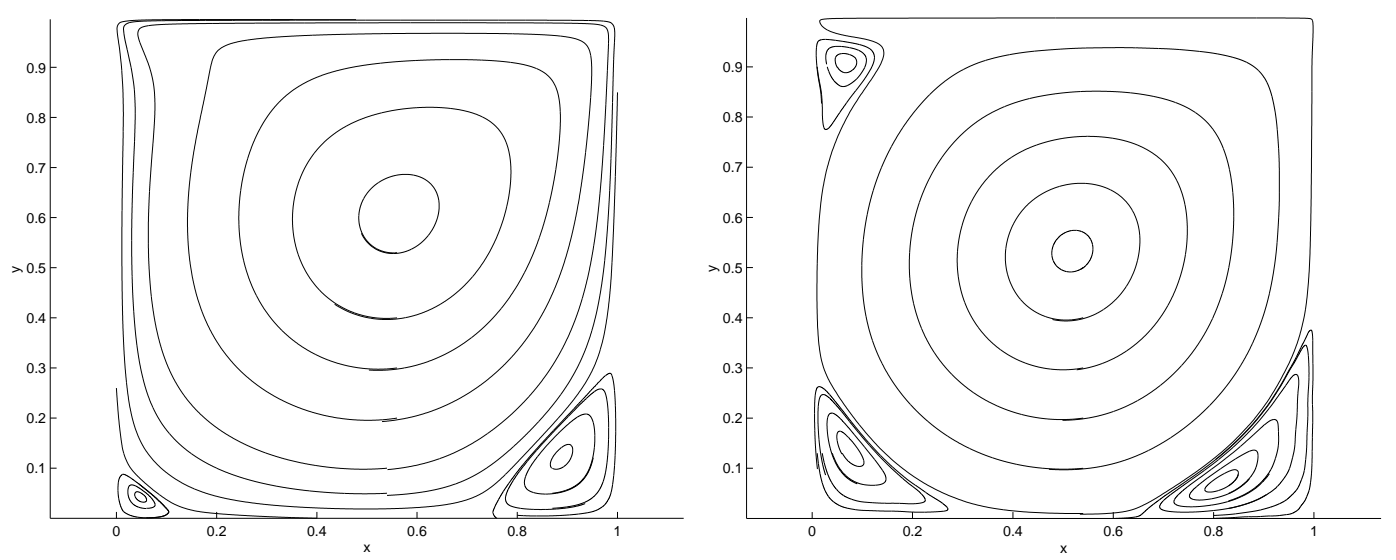

Figure 4: Streamlines of the computed solution at $R e=400$ (left) and $R e=5000$ (right).

The velocities are computed at $R e=5000$ on two grids with $\Delta x$ and $\Delta y$ increasing from the walls toward the center of the cavity. They are compared with the second order solutions in [15] on a vertical $(u)$ and a horizontal $(v)$ line through the geometrical midpoint. The results on the two grid lines in our grid closest to the midpoint are displayed in Figs. 5 and 6 . The improvement on the grid with half the step size is barely visible indicating that $41 \times 41$ grid points suffice for a well resolved solution. The results almost coincide with the values in [15] where the grid has $129 \times 129$ equidistantly distributed points. 

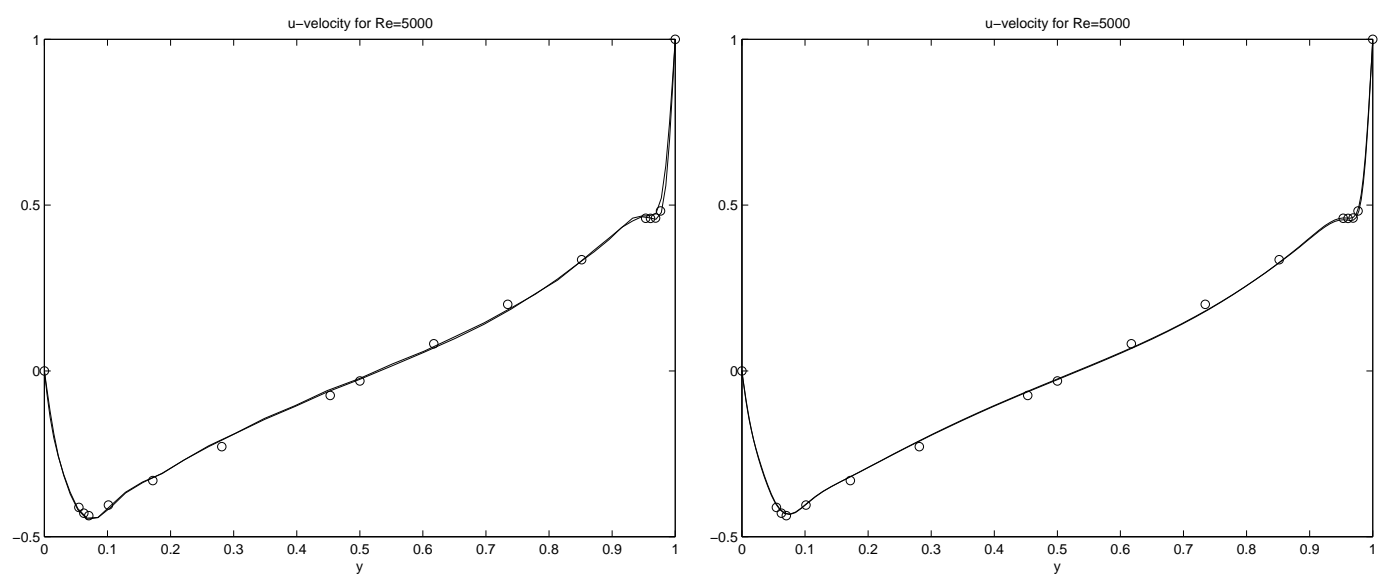

Figure 5: The computed solution for $u$ at $R e=5000$ (solid lines) is compared with results from [15] (o) along $x=0.5$ on a $41 \times 41$ grid (left) and on a $81 \times 81$ grid (right).
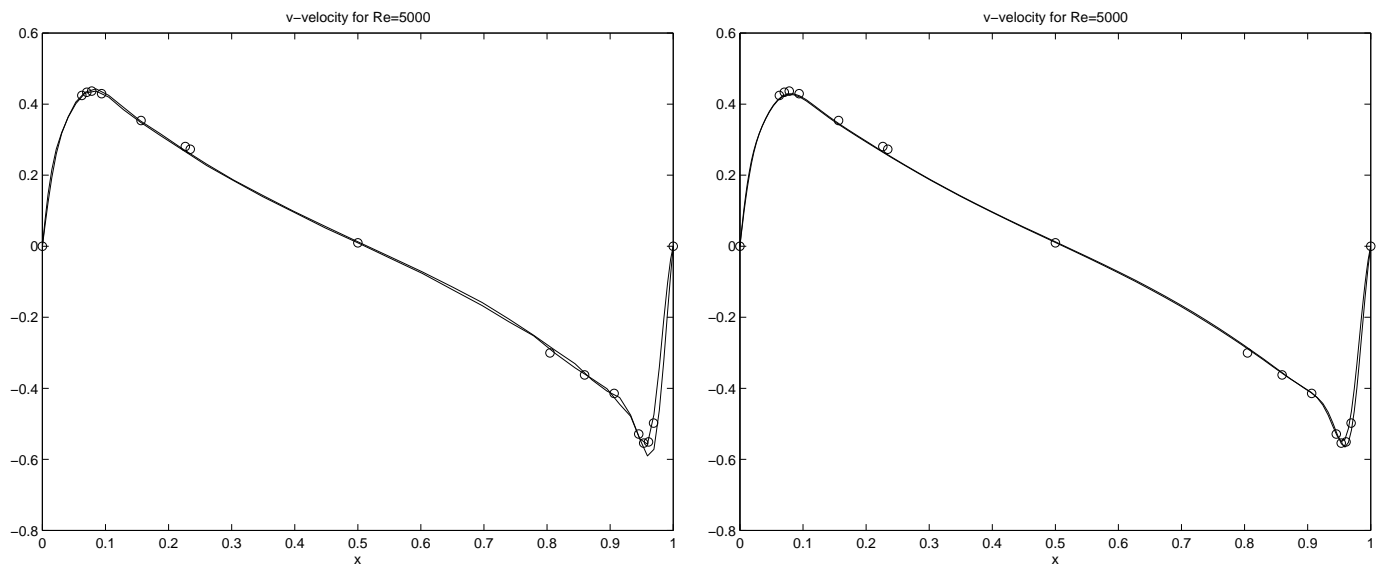

Figure 6: The computed solution for $v$ at $R e=5000$ (solid lines) is compared with results from [15] $(o)$ along $y=0.5$ on a $41 \times 41$ grid (left) and on a $81 \times 81$ grid (right).

\subsection{Forced solution in a constricting channel}

The flow is computed in a curvilinear grid covering a constricting channel of the same geometry as in [29]. The method in [29] is of fourth order in space for the streamfunction-vorticity formulation of Navier-Stokes' equations.

The transformation (5) from the Cartesian equidistant computational space $(\xi, \eta)$ to the physical curvilinear channel geometry is performed by a conformal mapping. This mapping $z=\mathcal{Z}(\zeta)$ between the computational $(\zeta=\xi+i \eta)$ and 
the physical space $(z=x+i y)$ is here computed analytically by the relation

$$
z=\zeta(A+B \tanh (\zeta))
$$

or in component form

$$
x=A \xi+\frac{B}{H}[\xi \sinh (2 \xi)-\eta \sin (2 \eta)], y=A \eta+\frac{B}{H}[\eta \sinh (2 \xi)+\xi \sin (2 \eta)],
$$

where $H=\cosh (2 \xi)+\cos (2 \eta)$ as in [29]. The channel width far away from the constriction is $2 a$ at the inlet and $2 b$ at the outlet. The constants $A$ and $B$ are defined as

$$
A=\frac{a+b}{2 \lambda}, \quad B=\frac{b-a}{2 \lambda},
$$

where the shape factor $\lambda$ controls the smoothness of the constriction.

The geometry in this experiment is a channel with $a=1$ and $b=0.7$ in (41), see Fig. 7. The boundary conditions of Dirichlet type (11) are chosen such that

$$
\begin{aligned}
& u=-\cos (x) \sin (y) \exp (-2 t / R e) \\
& v=\sin (x) \cos (y) \exp (-2 t / R e) \\
& p=-\frac{1}{4}(\cos (2 x)+\sin (2 y)) \exp (-4 t / R e)
\end{aligned}
$$

is a 'twilight-zone flow' solution [22] with a suitable forcing right hand side in the momentum equation (1). The same solution is chosen in [40]. The velocity is divergence free in (42). The solution for $R e=100$ is computed on grids with different resolution and compared with the exact solution (42). The spatial accuracy is evaluated as in (38) and the Tables 1, 2 and 3. The timestep $\Delta t=$ $10^{-5}$ is small to let the space error dominate.

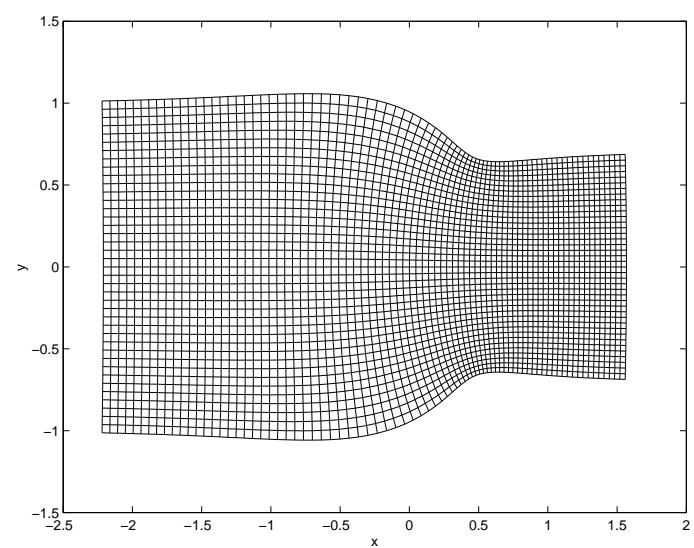

Figure 7: Grid of the constricting channel geometry .

The error $e_{k}$ on three grids $k=0,1,2$, with grid size $2^{k} h$ is evaluated and the order $q$ is computed by comparing $e_{k}, k=0,1$, with $e_{2}$ in Table 5 . 


\begin{tabular}{ccccc}
\hline \multirow{2}{*}{ Time step } & \multicolumn{3}{c}{ Maximum error and order } \\
\cline { 3 - 5 } & & $41 \times 21$ & $81 \times 41$ & $161 \times 81$ \\
\hline \multirow{2}{*}{600} & $u$ & $7.59 e-05$ & $4.34 e-06(4.1)$ & $2.41 e-07(4.2)$ \\
& $v$ & $1.67 e-04$ & $7.42 e-06(4.5)$ & $2.43 e-07(4.9)$ \\
& $p$ & $2.85 e-04$ & $1.56 e-05(4.2)$ & $7.94 e-07(4.3)$ \\
\hline \multirow{3}{*}{1000} & $u$ & $7.83 e-05$ & $4.81 e-06(4.0)$ & $3.00 e-07(4.0)$ \\
& $v$ & $1.66 e-04$ & $7.32 e-06(4.5)$ & $2.41 e-07(4.9)$ \\
& $p$ & $2.84 e-04$ & $1.55 e-05(4.2)$ & $8.16 e-07(4.3)$ \\
\hline
\end{tabular}

Table 5: Spatial errors and convergence rates measured in the $\ell_{2}$ norm of the forced solution in the constricting channel at $R e=100$

\subsection{Unforced solution in a constricting channel}
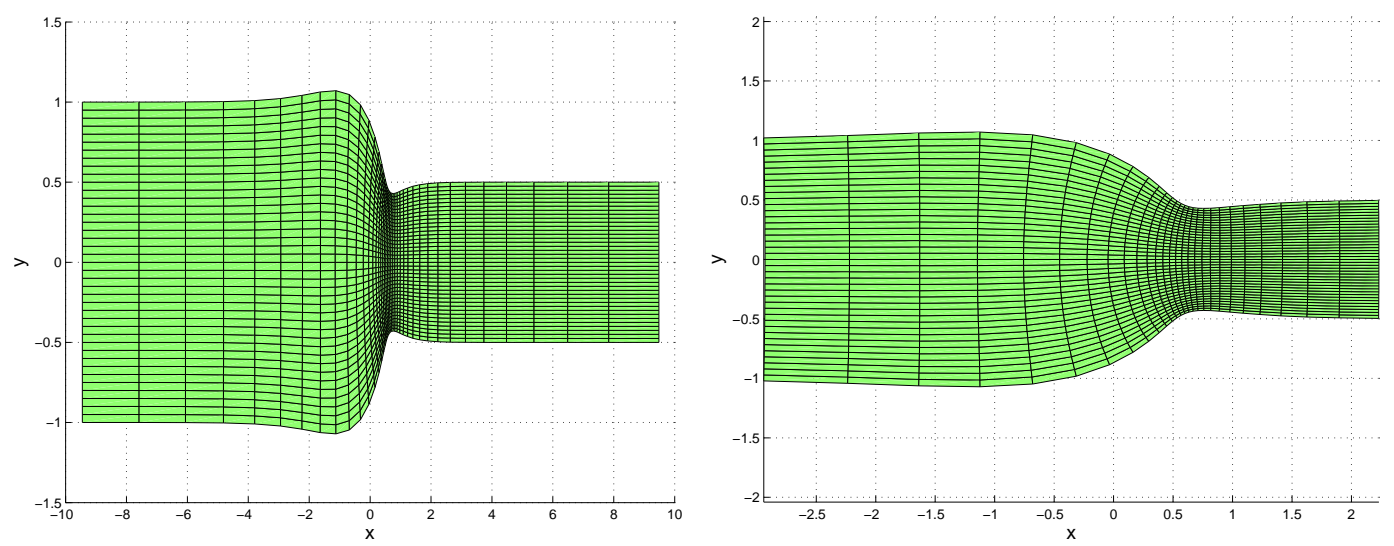

Figure 8: Grid of the constricting channel geometry. The full computational domain (left) and the area around the constriction (right) with equal scaling of the axes.

A constricting channel with a shape factor $\lambda=0.7$ and a ratio between the outlet and inlet heights of $b / a=0.7$ in (41) is constructed with the geometry depicted in Fig. 8. The grid is stretched in the streamwise direction before the conformal mapping (39), (40), is applied in order to move the inlet and outlet farther away from the constriction. We use the formula

$$
\xi\left(\xi_{1}\right)=C_{1} \sinh \left(C_{2} \xi_{1}\right)+\xi_{0}
$$

where $C_{1}=\lambda / 2, C_{2}=2 / \lambda$ and $\xi_{0}$ is the location of the singular point of the conformal mapping [29]. The flow is laminar without separation at $R e=150$ based on half the channel width. The no-slip condition is applied on the upper 
and lower walls and a Poiseuille profile is given at the inflow boundary. The outflow conditions are as in (12).

In the absence of an exact reference solution, the steady state solution is computed on four different grids with resolution $2^{k} h, k=0,1,2,3$, and $81 \times 81(k=$ $0), 41 \times 41(1), 21 \times 21(2)$, and $11 \times 11(3)$ grid points. The error in a variable $f_{k}$ on grid $k$ behaves as in (38) and the order of accuracy is estimated by the following two relations

$$
\frac{f_{3}-f_{2}}{f_{2}-f_{1}} \approx 2^{q_{1}}, \quad \frac{f_{2}-f_{1}}{f_{1}-f_{0}} \approx 2^{q_{2}}
$$

from which we can compute two spatial convergence rates $q_{1}$ and $q_{2}$. The spatial error can be estimated on the finest grid by assuming that $q=4$ and eliminating $f$ in (38). Then

$$
e_{0} \approx \frac{1}{15}\left(f_{1}-f_{0}\right)
$$

Due to the strong stretching in the streamwise coordinate, spatial convergence is investigated in the region around the constriction only, shown in the right panel of Fig. 8.

The error in the $\ell_{2}$ norm on the finest grid (45) and the convergence rates (44) are summarized in Table 6. The error behaves as expected with the best order of accuracy $q_{2}$ between the finest grids where the asymptotic regime of the error expansion in $h$ is reached.

\begin{tabular}{cccc}
\hline \multirow{2}{*}{ component } & \multicolumn{2}{c}{ convergence rate $(q$-order $)$} & \multirow{2}{*}{ error norm } \\
\cline { 2 - 4 } & $q_{1}$ & $q_{2}$ & \\
\hline$u$ & 3.33 & 4.02 & $9.78 e-6$ \\
$v$ & 2.92 & 3.45 & $1.04 e-5$ \\
$p$ & 2.65 & 4.18 & $1.92 e-5$ \\
\hline
\end{tabular}

Table 6: Spatial convergence rates and estimates of the numerical error on $81 \times 81$ grid measured in the $\ell_{2}$ norm for laminar flow in the constricting channel at $R e=150$

The convergence history of the outer and inner iterations (22), (24) and (25) is presented in Fig. 9. The performance is displayed for a long time step and a strict tolerance and a short time step and a tolerance at about $1 / 2$ of the accuracy in the solution in Table 6 . The initial solution in the interior is the constant zero solution. This is then integrated for the next 250 steps with Dirichlet inflow conditions and Neumann outflow conditions on the velocity (12). The number of outer iterations is 1 in most of the time steps. The solution of (24) is less efficient 
for a smaller $\varepsilon$ and a larger $\Delta t$ as expected from (30). The most time consuming part is the solution of the Poisson-like equation (25). This part is not sensitive to changes in $\Delta t$ and the increase in number of iterations from the left to the right figure in Fig. 9 is caused by the decrease in $\varepsilon$. To improve the efficiency of the method, the focus should be on reducing the computing time spent on the solution of (25).
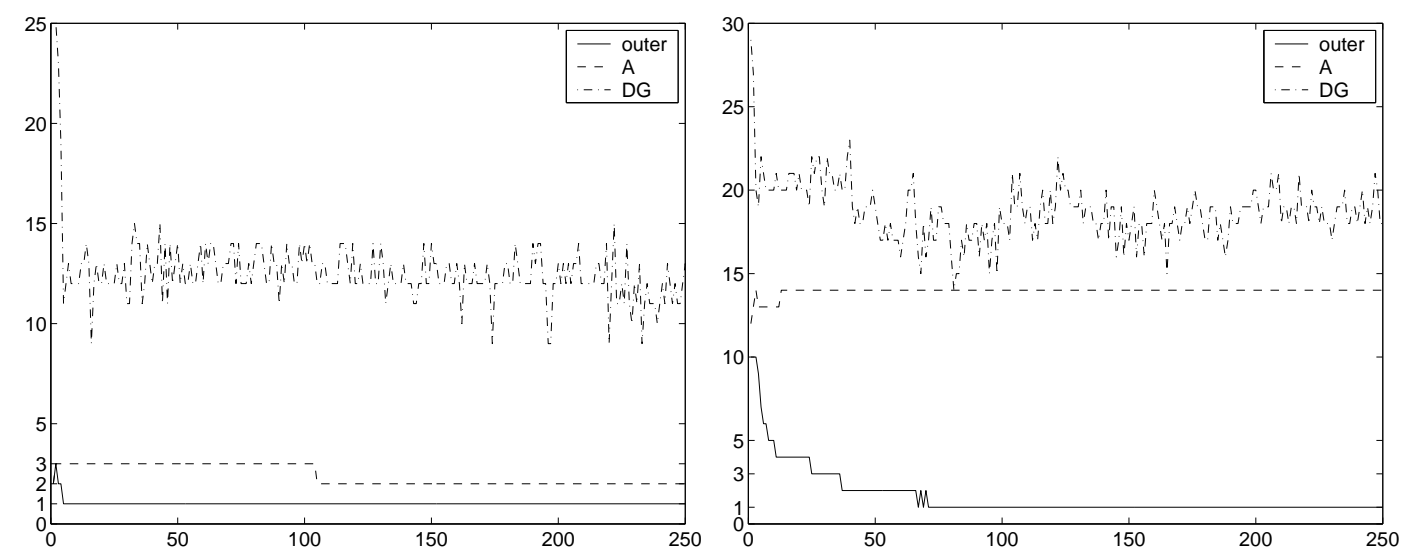

Figure 9: Convergence of the iterative method in the constricting channel with a $41 \times 41$ grid in each time step. The parameters are $\Delta t=0.001, \varepsilon_{o}=\varepsilon_{i}=5 \cdot 10^{-6}$, (left), and $\Delta t=0.005, \varepsilon_{o}=\varepsilon_{i}=5 \cdot 10^{-9}$, (right).

\section{Conclusions}

An accurate discretization of the incompressible Navier-Stokes equations in the primitive variables has been developed for two space dimensions. The method can be extended in the third dimension by a spectral approximation without too much difficulty. It is of fourth order accuracy in space and of second order in time in all variables. These orders have been verified in numerical experiments including curvilinear grids. The boundary conditions have been proved to be stable for Cartesian grids and are so in practice also for non-Cartesian grids. The compact difference operators simplify the treatment at the numerical boundaries and no extra numerical boundary conditions are needed for the pressure. The variables are located in a staggered grid to improve the accuracy and to make the method less prone to oscillatory behavior. A system of linear equations is solved in every time step using outer and inner iterations with preconditioning of the subproblems. The difficulty with the nonuniqueness of the pressure and a singular system matrix is avoided by the definition of the boundary conditions. The outer iterations are preconditioned by an approximate factorization with control of the iteration errors. 


\section{Acknowledgment}

Financial support has been obtained from the Swedish Research Council for Engineering Science and the Swedish Research Council. We grateful to Professors Dan Henningson and Arne Johansson for helping us with their profound knowledge in flow physics.

\section{References}

[1] O. Axelsson, Iterative Solution Methods, Cambridge Univ. Press, Cambridge, 1994.

[2] K. Bhaganagar, D. Rempfer, J. Lumley, Direct numerical simulation of spatial transition to turbulence using fourth-order vertical velocity secondorder vertical vorticity formulation, J. Comput. Phys., 180 (2002), 200-228.

[3] D. L. Brown, R. Cortez, M. L. Minion, Accurate projection methods for the incompressible Navier-Stokes equations, J. Comput. Phys., 168 (2001), 464-499.

[4] A. BRÜGER, Higher order methods suitable for direct numerical simulation of flows in complex geometries, Licentiate thesis, Dept of Mechanics, Royal Institute of Techonolgy, Stockholm, Sweden, 2002.

[5] A. Brüger, J. Nilsson, W. Kress, A compact higher order finite difference method for the incompressible Navier-Stokes equations, J. Sci. Comput., 17 (2002), 551-560.

[6] J. Cahouet, J.-P. Chabard, Some fast 3D finite element solvers for the generalized Stokes problem, Int. J. Numer. Meth. Fluids, 8 (1988), 869-895.

[7] W. Chang, F. Giraldo, B. Perot, Analysis of an exact fractional step method, J. Comput. Phys., 180 (2002), 183-199.

[8] H. Chor, P. Moin, Effects of the computational time step on numerical solutions of turbulent flow, J. Comput. Phys., 113 (1994), 1-4.

[9] J. K. Dukowicz, A. S. Dvinsky, Approximate factorization as a high order splitting for the implicit incompressible flow equations, J. Comput. Phys., 102 (1992), 336-347.

[10] H. C. Elman, Preconditioners for saddle point problems arising in computational fluid dynamics, Appl. Numer. Math., 43 (2002), 75-89. 
[11] H. C. Elman, V. E. Howle, J. N. Shadid, R. S. Tuminaro, A parallel block multi-level preconditioner for the 3D incompressible Navier-Stokes equations, J. Comput. Phys., 187 (2003), 504-523.

[12] H. C. Elman, D. J. Silvester, A. J. Wathen, Performance and analysis of saddle point preconditioners for the discrete steady-state Navier-Stokes equations, Numer. Math., 90 (2002), 665-688.

[13] B. Fornberg, M. Ghrist, Spatial finite difference approximations for wave-type equations, SIAM J. Numer. Anal., 37 (1999), 105-130.

[14] R. Friedrich, T. J. Hüttr, M. Manhart, C. Wagner, Direct numerical simulation of incompressible turbulent flows, Computers \& Fluids, 30 (2001), 555-579.

[15] U. Ghia, K. N. Ghia, C. T. Shin, High Re solutions for incompressible flow using the Navier-Stokes equation and multigrid methods, J. Comput. Phys., 48 (1982), 387-411.

[16] S. Ghosal, An analysis of numerical errors in large-eddy simulations of turbulence, J. Comput. Phys., 125 (1996), 187-206.

[17] A. Greenbaum, Iterative Methods for Solving Linear Systems, SIAM, Philadelphia, 1997.

[18] B. Gustafsson, P. Lötstedt, A. Göran, A fourth order difference method for the incompressible Navier-Stokes equations, in M.M. Hafez, editor, Numerical simulations of incompressible flows, World Scientific Publishing, Singapore, 2003, 263-276.

[19] B. Gustafsson, J. Nilsson, Boundary conditions and estimates for the steady Stokes equations on staggered grids, J. Sci. Comput., 15 (2000), 2954 .

[20] B. Gustafsson, J. Nilsson, Fourth order methods for the Stokes and Navier-Stokes equations on staggered grids, in D. A. Caughey, M. M. Hafez, editors, Frontiers of Computational Fluid Dynamics - 2002, World Scientific Publishing, Singapore, 2002, 165-179.

[21] E. Hairer, S. P. Nørsett, G. Wanner, Solving Ordinary Differential Equations I, Nonstiff Problems, 2nd ed., Springer-Verlag, Berlin, 1993.

[22] W. D. Henshaw, A fourth-order accurate method for the incompressible Navier-Stokes equations on overlapping grids, J. Comput. Phys., 113 (1994), $13-25$. 
[23] G. E. Karniadakis, M. Israeli, S. A. Orszag, High-order splitting methods for incompressible Navier-Stokes equations, J. Comput. Phys., 97 (1991), 414.

[24] J. Kim, P. Moin, Application of a fractional-step method to incompressible Navier-Stokes equations, J. Comput. Phys., 59 (1985), 308-323.

[25] W. Kress, J. Nilsson, Boundary conditions and estimates for the linearized Navier-Stokes equations on a staggered grid, Computers \& Fluids, 32 (2003), 1093-1112.

[26] S. K. LELE, Compact finite difference schemes with spectral-like resolution, J. Comput. Phys., 103 (1992), 16-42.

[27] M. Li, T. TAng, B. Fornberg, A compact fourth-order finite difference scheme for the steady incompressible Navier-Stokes equations, Int. J. Numer. Meth. Fluids, 20 (1995), 1137-1151.

[28] A. Lundbladh, D. S. Henningson, A. V. Johansson, An efficient spectral integration method for the solution of the time-dependent NavierStokes equations, Report FFA-TN 1992-28, Aeronautical Research Institute of Sweden, Bromma, Sweden, 1992.

[29] P. F. De A. Mancera, R. Hunt, Fourth order method for solving the Navier-Stokes equations in a constricting channel, Int. J. Numer. Meth. Flu$i d s, 25$ (1997), 1119-1135.

[30] J. A. Meijerink, H. A. VAn Der Vorst, An iterative solution method for linear systems of which the coefficient matrix is a symmetric M-matrix, Math. Comp., 31 (1977), 148-162.

[31] R. S. Montero, I. M. Llorente, M. D. Salas, Robust multigrid algorithms for the Navier-Stokes equations, J. Comput. Phys., 173 (2001), 412-432.

[32] Y. Morinishi, T. S. Lund, O. V. Vasilyev, P. Moin, Fully conservative higher order finite difference schemes for incompressible flow, J. Comput. Phys., 143 (1998), 90-124.

[33] S. Nagarajan, S. K. Lele, J. H. Ferziger, A robust high-order compact method for large eddy simulation, J. Comput. Phys., 191 (2003), 392419.

[34] J. Nilsson, Initial-boundary-value problems for the Stokes and NavierStokes equations on staggered grids, PhD thesis, Dept of Scientific Computing, Uppsala University, Uppsala, Sweden, 2000. 
[35] M. Ofstad Henriksen, J. Holmen, Algebraic splitting for incompressible Navier-Stokes equations, J. Comput. Phys., 175 (2002), 438-453.

[36] M. Pernice, M. D. Tocci, A multigrid-preconditioned Newton-Krylov method for the incompressible Navier-Stokes equations, SIAM J. Sci. Comput., 23 (2001), 398-418.

[37] J. B. Perot, An analysis of the fractional step method, J. Comput. Phys., 108 (1993), 51-58.

[38] N. A. Petersson, Stability of pressure boundary conditions for Stokes and Navier-Stokes equations, J. Comput. Phys., 172 (2001), 40-70.

[39] P. J. Schmid, D. S. Henningson, Stability and Transition in Shear Flows, Springer, New York, 2001.

[40] J. C. STRIKWERDA, High-order-accurate schemes for incompressible viscous flow, Int. J. Numer. Meth. Fluids, 24 (1997), 715-734.

[41] J. C. Strikwerda, Y. S. Lee, The accuracy of the fractional step method, SIAM J. Numer. Anal., 37 (1999), 37-47.

[42] S. Turek, A comparative study of time-stepping techniques for the incompressible Navier-Stokes equations: From fully implicit non-linear schemes to semi-implicit projection methods, Int. J. Numer. Meth. Fluids, 37 (1999), $37-47$.

[43] A. Veneziani, Block factorized preconditioners for high-order accurate in time approximation of the Navier-Stokes equations, Numer. Meth. Part. Diff. Eq., 19 (2003), 487-510.

[44] H. A. VAn DER Vorst, BiCGSTAB: A fast and smoothly converging variant of Bi-CG for the solution of nonsymmetric linear systems, SIAM J. Sci. Comput., 13 (1992), 631-644. 